\title{
Un programa de prevención temprana de la obesidad: “HaViSa UC" (2009-2019)
}

\author{
An Early Obesity Prevention Program: HaViSa UC (2009-2019)
}

\author{
Barja $S^{a}$, Loyola $M^{b}$, Ortiz $C^{b}$, Araneda $Y^{b}$, Undurraga $R^{c}$.
}

aMédico. Magíster en Nutrición Pediátrica. Departamento de Gastroenterología y Nutrición Pediátrica. División de Pediatría, Facultad de Medicina. Pontificia Universidad Católica de Chile. Santiago, Chile

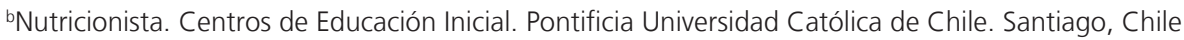

'Educadora de Párvulos. Jefe del Departamento de Beneficios al Personal. Pontificia Universidad Católica de Chile. Santiago, Chile

Recibido: 10 de diciembre de 2019; Aceptado: 16 de marzo de 2020

¿Qué se sabe del tema que trata este estudio?

La prevención es la solución definitiva de la obesidad y debe realizarse a lo largo de todo el ciclo vital, pero programas preventivos de corta a mediana duración no han logrado mejoría significativa del estado nutricional de niños y adolescentes.

\section{¿Qué aporta este estudio a lo ya conocido?}

A 10 años de su implementación, este programa de estímulo de hábitos saludables aplicado a lactantes y preescolares en salas cuna y jardines infantiles, disminuyó la prevalencia de la obesidad y sobrepeso; Se requieren programas preventivos tempranos y permanentes.

\section{Resumen}

Introducción: La prevención es la solución definitiva al grave problema epidemiológico nutricional de la niñez en nuestro país y el mundo, que es la obesidad. Objetivo: Describir los resultados de un programa de prevención de la obesidad en lactantes y preescolares, a diez años de su inicio. Sujetos y Método: Estudio retrospectivo, cuasi experimental, de la prevalencia de sobrepeso y obesidad, en niños y niñas asistentes a las salas-cuna y jardines infantiles de la Universidad Católica de Chile, desde la implementación en 2009 de un programa de promoción precoz de hábitos de vida saludable en alimentación y actividad física (HaViSa-UC), hasta 2019. Se obtuvo aprobación ética y se analizaron los registros anuales de las evaluaciones antropométricas (referencia OMS 2006), de cada mes de marzo, en los centros de tres campus universitarios, utilizando el programa Minitab 17. Las acciones implementadas por el HaViSa-UC fueron: evaluación del estado nutricional y comunicación con los padres, entrega de una alimentación saludable, promoción de un hábito activo de vida y educación para favorecer hábitos saludables de vida. Resultados: El promedio anual fue de 319 asistentes, $14 \%$ menores de dos años y 49,5\% niñas. En marzo 2009 se detectó 32,6\% de sobrepeso y $8,6 \%$ de
Palabras clave:

Obesidad;

sobrepeso;

pediatría; prevención; vida saludable

Correspondencia:

Salesa Barja

sbarja@uc.cl 
obesidad, cifras que disminuyeron y se estabilizaron, alcanzando en marzo de 2019 a 23,8\% y 4,7\% respectivamente. Los eutróficos aumentaron desde 56,9\% a 67,4\%, sin aumentar el bajo peso. En el mismo período, $\mathrm{zP} / \mathrm{T}$ bajó desde $0,84 \pm 0,94$ a $0,55 \pm 0,87(\mathrm{p}=0,00)$, y zT/E aumentó desde $-0,36 \pm$ 0,87 a $-0,32 \pm 0,90(p>0,05)$. Conclusión: Desde la implementación del programa HaViSa, en esta muestra de lactantes y preescolares la frecuencia de obesidad bajó en 45,4\% y el sobrepeso en 27\%, con estabilidad al cabo de diez años.

\section{Abstract}

Introduction: Prevention is the definitive solution to the serious nutritional epidemiological problem of children in our country and the world, obesity. Objective: To describe the results of an obesity prevention program for infants and preschoolers, ten years after its implementation. Subjects and Methods: Retrospective, and quasi-experimental study of the overweight and obesity prevalence, in children attending three nursery and preschool centers located at the Universidad Católica de Chile, since the implementation of a multidimensional program for early promotion of healthy lifestyle habits (HaViSa-UC) between 2009 and 2019. This study obtained ethical approval. Annual records of anthropometric assessment (WHO 2006) were analyzed using Minitab 17 software. The actions applied by the HaViSa-UC program were the assessment of nutritional status and communication with parents, delivery of healthy food, promotion of an active lifestyle, and education to encourage such healthy habits. Results The annual mean was 319 subjects, $14 \%$ younger than two years old, and 49.5\% were girls. In March 2009 (baseline), 32.6\% had overweight and 8.6\% obesity; both figures decreased reaching $23.8 \%$ and $4.7 \%$ respectively, in March 2019. Normal weight increased from 56.9 to $67.4 \%$ and malnutrition presented no increase. In the same period, $\mathrm{zW} / \mathrm{H}$ dropped from $0.84 \pm$ 0.94 to $0.55 \pm 0.87$ (p: 0.00 ), and $\mathrm{zH} / \mathrm{A}$ increased from $-0.36 \pm 0.87$ to $-0.32 \pm 0.90$ (p $>0.05$ ). Conclusion: Since the implementation of the HaViSa-UC Program, the frequency of obesity decreased by $45.4 \%$ and overweight by $27.2 \%$ in this sample of infants and preschoolers, remaining stable after 10 years.

\section{Keywords:}

Obesity; overweight; pediatrics; prevention; healthy life

\section{Introducción}

La obesidad es el problema epidemiológico más grave, de mayor prevalencia y en ascenso, en la población chilena ${ }^{1-3}$, que se inicia tempranamente en la vida y tiende a perpetuarse en el tiempo ${ }^{4,5}$. Afecta a todo el organismo, con complicaciones a corto plazo como hipertrigliceridemia, trastornos psicológicos y del sueño, así como a mediano y largo plazo hipertensión arterial, hígado graso, diabetes tipo 2 o dislipidemias, entre otras ${ }^{6}$. Más aún, el tratamiento de la obesidad tiene bajo rendimiento ${ }^{7-9} \mathrm{y}$ mientras más tiempo permanezca, se asociará a peor calidad de vida y mayor morbimortalidad en la adultez ${ }^{10,11}$. El sobrepeso afecta a $24 \%$ y la obesidad a $11 \%$ de los menores de seis años que se encuentran bajo control en el sistema público de sa$\operatorname{lud}^{2}$. La prevalencia asciende desde los primeros meses de vida y se acentúa después de los dos años, superando en conjunto a la población eutrófica en escolares de primer año básico, que en 2018 presentaba 26,4\% de sobrepeso y $24,4 \%$ de obesidad ${ }^{3,12,13}$.

Frente a esta pandemia de obesidad y sus consecuencias, existe consenso en que la prevención es la principal y definitiva solución, con estrategias multidimensionales, implementadas de modo precoz, perma- nentes en el tiempo y a lo largo de todo el ciclo vital. La prevención se basa en la promoción universal de cambios perdurables en el estilo de vida, que consideren una alimentación saludable y balanceada, junto a un estilo activo de vida ${ }^{14-16}$. De este modo, aumentar los factores protectores y las conductas saludables desde la niñez temprana y mantenerlos posteriormente, es un objetivo fundamental en pro de una mejor salud cardiovascular ${ }^{17}$.

Chile ha logrado un avance importante en políticas públicas poblacionales contra la obesidad a través de la implementación de las leyes $n^{\circ} 20.605$ y 20.869 , que regulan la composición de los alimentos y la publicidad dirigida a la población infantil de aquellos no saludables ${ }^{18}$. Sin embargo, los esfuerzos deben realizarse en todos los sistemas que rodean al niño, como lo plantean los modelos ecológicos, que analizan en forma integral y transversal la prevención y tratamiento de los problemas epidemiológicos en la población $^{19,20}$.

Los mejores resultados de los programas para prevenir la obesidad se obtienen en base a cambios que involucran en conjunto la alimentación saludable y el estímulo de actividad física ${ }^{15,21-24}$, especialmente durante los primeros años, cuando los niños adquieren hábitos 
que serán progresivamente más difíciles de modificar con el paso del tiempo.

En Chile se han aplicado programas preventivos como el de Casablanca, durante dos años a escolares de $1^{\circ}$ a $7^{\circ}$ básico y/o profesores, logrando una disminución de la obesidad de 17 a 12,3\% en los hombres ${ }^{25}$. Otra experiencia, en niños de prekínder a $1^{\circ}$ básico no logró mejoría del estado nutricional al cabo de un año ${ }^{26}$. Sin embargo, no se dispone de reportes en niños de menor edad, ni de programas de mayor duración a la descrita.

El año 2009 se implementó el programa HaViSa UC (Hábitos de vida saludable en la UC) en tres centros de Educación Inicial de la Pontificia Universidad Católica de Chile, destinado al fomento precoz de hábitos saludables de alimentación y actividad física en lactantes y preescolares, para favorecer una mejor salud y prevenir la obesidad.

El objetivo del presente estudio fue describir los resultados de este programa, a diez años de su inicio.

\section{Sujetos y Método}

Se realizó un estudio de diseño longitudinal retrospectivo, cuasi- experimental, para evaluar los cambios en la prevalencia de obesidad y sobrepeso, antes y después de la implementación del programa HaViSa UC, entre los años 2009 y 2019.

Se incluyeron los datos de los niños asistentes a los tres centros de educación inicial de la UC. Cada centro se ubica cerca (Casa Central) o dentro (Campus Oriente y San Joaquín), de un campus universitario, donde asisten hijos de funcionarias de la Universidad y la Red de Salud UC Christus; corresponden a personal administrativo, académico y alumnas. La muestra estuvo conformada por todos los niños que estuvieron presentes durante los días en que se realizó la evaluación nutricional, en el mes de marzo de cada año. Se excluyeron los niños con información incompleta.

Se registraron las variables demográficas de edad (en meses) y género $(\mathrm{F} / \mathrm{M})$ y evaluaciones antropométricas realizadas por dos nutricionistas (CO, YA) de modo estandarizado.

Las variables incluidas fueron: peso (gramos), talla $(\mathrm{cm})$, puntajes $\mathrm{z}$ de índice peso/edad (zP/E), Talla/Edad (zT/E), Peso/Talla (zP/T) e índice de masa corporal (zIMC). Se definió los estados nutricionales según referencia OMS 2006 ${ }^{27}$ : obesidad $(\mathrm{zP} / \mathrm{T} \geq+2)$, sobrepeso $(\mathrm{zP} / \mathrm{T}+1$ a $+1,99)$, eutrofia $(\mathrm{zP} / \mathrm{T}-0,99$ a $+0,99)$, riesgo de desnutrición $(-1$ a $-1,99)$ y desnutrición $(\leq-2)$. Para estos dos últimos, se usó zP/E en menores de un año y zP/T en mayores de un año. Se consideró el antecedente de prematurez para corregir la edad cronológica y se usaron curvas específicas en niños con síndrome de Down ${ }^{44}$.
Se registró la edad y educación materna como aproximación al nivel socioeconómico (NSE). La edad materna en 2009 fue de 34,6 \pm 5,4 años, con nivel educativo de Educación Media (10,9\%), Técnico profesional $(45,5 \%)$ o universitaria $(43,6 \%)$. La jornada de permanencia de los niños en los centros fue completa en el 99,9\% de los casos.

\section{Descripción del Programa HaViSa UC}

Las acciones implementadas gradualmente en todos los centros fueron:

\section{Evaluación del estado nutricional y comunica- ción con los padres:}

Se estableció una entrevista a los padres al ingreso, con encuesta de hábitos de alimentación y actividad física. Se actualizaron los instrumentos de medición y se instauró la evaluación antropométrica en marzo, julio y diciembre, con envío de un informe impreso a los padres, del estado nutricional y su evolución e invitación a entrevista al detectar malnutrición por exceso o déficit. Todo lo anterior realizado por la nutricionista.

\section{Alimentación saludable:}

Se acentuó la promoción de lactancia materna $(\mathrm{LM})^{28-31}$, se elaboró un registro de lactantes amamantados, una guía para extracción y envío de LM, junto a un reglamento de conservación, manipulación y administración en el centro. Se adaptó una sala de lactancia en cada centro según el manual de lactancia MINSAL 2000 y a partir de 2017, se habilitaron según la Guía de implementación de MINSAL de ese mismo año.

Para la entrega de una alimentación inocua, se realizó un diagnóstico y se actualizó la planta física de los servicios de alimentación y SEDILES (RSA. DS 977/96), se elaboró un manual de prácticas seguras, con monitoreo regular del cumplimiento y evaluación anual de exámenes de laboratorio del personal de alimentación.

Se realizó una evaluación basal de calidad y cantidad de la alimentación complementaria y se elaboraron minutas acordes a las recomendaciones de FAOOMS 2000, la Guía de alimentación infantil MINSAL 2007 y posteriormente su actualización de 2016, con énfasis en su aplicación en comidas tradicionales ${ }^{32-35}$. Se logró adaptación gradual y buen cumplimiento. Se publicaron informes mensuales en el diario mural y registro de ingesta en la agenda del niño.

Se reemplazaron jugos comerciales azucarados por agua de cocción de frutas y posteriormente, por agua fresca a través de dispensadores ${ }^{36,37}$. Se homogeneizó el tipo y concentración de las fórmulas lácteas, adaptadas al estado nutricional, en aquellos con exceso de peso: sin adición de cereal en lactantes de 6 a 12 meses, fór- 
mula semidescremada en 12 a 24 meses, o descremada en los mayores de 2 años $^{38}$. Se reemplazaron las colaciones procedentes del hogar por una fruta fresca entregada por el centro, y se envió pauta de cumpleaños saludables a los padres.

Se promovió un estilo respetuoso de alimentación, con participación infantil individual y grupal ${ }^{32}$. Frente al rechazo alimentario, se suspendió el reemplazo por lácteo o alternativa.

\section{Promoción de un hábito activo de vida ${ }^{40-42}$ :}

Se disminuyó la duración de la siesta de 120 a 90 minutos diarios en los lactantes o del tiempo de descanso en litera en los mayores. Se suspendió el uso de películas de entretención y se aumentó el juego activo a 15-20 minutos por día, según nivel. En los mayores de 2 años, se instauró una clase semanal de 30 minutos, realizada por un profesor de Educación física.

\section{Educación para favorecer hábitos saludables de vida:}

Las actividades educativas para los niños incluyeron sesiones de juego con contenidos clave de consumo de agua, de frutas y verduras ${ }^{32}$, comida en familia ${ }^{39}$ y estructura de la alimentación (tiempos y calidad). Para envío al hogar, se implementaron fichas, calendario de frecuencias y actualmente, un individual o placemat para colorear, con las porciones de un almuerzo saludable. Se añadieron talleres de preparación de frutas, taller con el chef y visita semanal al huerto en un centro. También sesiones sobre juego activo (caminar, correr y saltar, juegos y rondas infantiles), folklore, deportes y menor tiempo de pantallas ${ }^{43}$.

Para los padres, se elaboró un video de presentación del programa HaViSa, para la primera reunión del año, complementario a la información incluida en el reglamento del Jardín. Se elaboraron contenidos educativos adosados al reporte de evaluación nutricional: en marzo (Guías anticipatorias), en julio (Guía de composición de la alimentación y frecuencia de consumo) y en diciembre (recomendaciones para vacaciones y de egreso). Además se elaboró un recetario de las comidas que reciben los niños en el jardín (diciembre de 2019).

Además, se organizaron actividades educativas y de capacitación, dos veces al mes para el personal del servicio de alimentación y una vez al mes para educadoras y personal técnico, sobre seguridad alimentaria, trastornos de alimentación, alergias, y otras establecidas de común acuerdo.

\section{Análisis estadísticos}

Se describieron las prevalencias de los distintos estados nutricionales para cada evaluación anual entre los años 2009 y 2019. Se analizó la distribución de los puntajes z para los índices antropométricos con test de Ryan-Joiner y se describieron los promedios y D.E. para cada evaluación anual. Se estudiaron las diferencias según sexo, edad y centro con test de ANOVA para variables numéricas (con test post hoc de Fisher y Tukey) y test de Chi2 para las categóricas. Se compararon las prevalencias de sobrepeso y obesidad $\mathrm{HaViSa}$ 2019 con las reportadas a nivel nacional, mediante el test de una proporción (IC 95\%). El nivel exigido de significación fue $\mathrm{p}<0,05$ y se utilizó el programa Minitab17.

\section{Aspectos éticos}

Este estudio se realizó respetando las normas éticas establecidas por la Declaración de Helsinki (1975 y revisada en 1983) y obtuvo aprobación del Comité de ética en investigación de la Facultad de Medicina, Universidad Católica de Chile, (\#15-007), obteniéndose dispensa de consentimiento informado. Se realizó información del programa a través del reglamento que se entrega a los padres al ingreso de sus hijos a los centros, el mes de marzo de cada año. Se respetó la privacidad de los datos sensibles para los registros de las bases y los análisis estadísticos realizados.

\section{Resultados}

En la tabla 1 se describen las características principales de la muestra de cada mes de marzo durante los diez años del programa. El tamaño muestral fue similar, con una proporción constante de mujeres de $49,2 \%$. En cuanto a la edad, se observaron promedios similares, con una proporción de $14,1 \%$ de menores de 12 meses, $30,4 \%$ de 12 a 24 meses, $27,8 \%$ de 2 a 3 años y $27,7 \%$ de mayores de 3 años, que se mantuvo estable en el tiempo.

En la figura 1 se observa la evolución de las prevalencias de los estados nutricionales en el mes de marzo de cada año, con una caída inicial de la obesidad desde $8,6 \%$ a $3,5 \%$ en 2013 , seguida por elevación leve y fluctuaciones menores, hasta $4,7 \%$ en 2019 ( $p=0,038)$. El sobrepeso disminuyó desde $32,7 \%$ a $24 \%$, y se mantuvo estable hasta $23,8 \%$ en 2019 ( $p=0,000)$. La malnutrición por exceso, por tanto, disminuyó globalmente desde $41,2 \%$ a $28,5 \%(p=0,000)$. Durante estos diez años, la malnutrición por déficit fluctuó entre 1,1\% y $8,8 \%$, grupo conformado por niños con riesgo a desnutrir, de predominio menores de 12 meses, y sólo un caso de desnutrición. Globalmente, la proporción de eutróficos aumentó desde 56,9\% a 67,4\%.

La proporción de talla baja osciló entre 1,56 y $5,05 \%$ y la de talla alta, entre 0 y $3,44 \%$.

$\mathrm{El} \mathrm{zP/T}$ tuvo un descenso mayor los primeros 4 años del programa, con leve ascenso y estabiliza- 
ción posterior; globalmente, entre 2009 y 2019, bajó desde $0,84 \pm 0,94$ a $0,55 \pm 0,87(\mathrm{p}=0,0004)$, con diferencia de $-0,29 \pm 0,07(-0,43 ;-0,15), \mathrm{p}=0,000$. $\mathrm{El} \mathrm{zT/E}$ aumentó desde $-0,36 \pm 0,87$ a $-0,32 \pm 0,90)$, con diferencia de $0,036 \pm 0,08(-0,12 ; 0,19) \mathrm{p}>0,05$. La tabla 2 muestra los promedios para cada año de ambos índices antropométricos y la figura 2 grafica su evolución.

Tabla 1. Características de los lactantes y preescolares asistentes a los jardines infantiles PUC entre 2009 y 2019

\begin{tabular}{|c|c|c|c|c|c|c|c|c|c|c|c|}
\hline Año & 2009 & 2010 & 2011 & 2012 & 2013 & 2014 & 2015 & 2016 & 2017 & 2018 & 2019 \\
\hline$n$ & 267 & 259 & 297 & 320 & 319 & 336 & 319 & 347 & 370 & 357 & 319 \\
\hline $\begin{array}{l}\text { Edad } \\
\text { (meses) }^{1}\end{array}$ & $\begin{array}{c}27,7 \\
\pm 14,8\end{array}$ & $\begin{array}{l}28,7^{A} \\
\pm 14,1\end{array}$ & $\begin{array}{l}29,1^{\mathrm{A}} \\
\pm 14,8\end{array}$ & $\begin{array}{r}27,9 \\
\pm 14,7\end{array}$ & $\begin{array}{c}27,7 \\
\pm 13,6\end{array}$ & $\begin{array}{r}27,9 \\
\pm 13,8\end{array}$ & $\begin{array}{l}25,4^{\mathrm{D}} \\
\pm 11,7\end{array}$ & $\begin{array}{l}25,4^{\mathrm{D}} \\
\pm 12,4\end{array}$ & $\begin{array}{r}25,3^{\mathrm{D}} \\
\pm 11,9\end{array}$ & $\begin{array}{r}25,9 \\
\pm 11,1\end{array}$ & $\begin{array}{r}26,2 \\
\pm 11,9\end{array}$ \\
\hline Mujeres (\%) & 48,7 & 51,0 & 50,8 & 48,7 & 49,8 & 50,3 & 47,3 & 51,6 & 48,2 & 47,9 & 50,5 \\
\hline $\begin{array}{l}\text { Prematuros } \\
\text { (\%) }\end{array}$ & $\begin{array}{c}11 \\
(4,1)\end{array}$ & $\begin{array}{c}13 \\
(5,0)\end{array}$ & $\begin{array}{c}8 \\
(2,7)\end{array}$ & $\begin{array}{c}10 \\
(3,1)\end{array}$ & $\begin{array}{c}1 \\
(0,3)\end{array}$ & $\begin{array}{c}4 \\
(1,2)\end{array}$ & $\begin{array}{c}2 \\
(0,6)\end{array}$ & $\begin{array}{c}6 \\
(1,7)\end{array}$ & $\begin{array}{c}10 \\
(2,7)\end{array}$ & $\begin{array}{c}8 \\
(2,2)\end{array}$ & $\begin{array}{c}7 \\
(2,2)\end{array}$ \\
\hline $\begin{array}{l}\text { S. Down } \\
(\%)\end{array}$ & $\begin{array}{c}0 \\
(0)\end{array}$ & $\begin{array}{c}1 \\
(0,4)\end{array}$ & $\begin{array}{c}1 \\
(0,3)\end{array}$ & $\begin{array}{c}1 \\
(0,3)\end{array}$ & $\begin{array}{c}1 \\
(0,3)\end{array}$ & $\begin{array}{c}3 \\
(0,9)\end{array}$ & $\begin{array}{c}1 \\
(0,3)\end{array}$ & $\begin{array}{c}3 \\
(0,9)\end{array}$ & $\begin{array}{c}3 \\
(0,8)\end{array}$ & $\begin{array}{c}1 \\
(0,28)\end{array}$ & $\begin{array}{c}1 \\
(0,3)\end{array}$ \\
\hline $\begin{array}{l}\text { Gemelos } \\
(\%)\end{array}$ & $\begin{array}{c}6 \\
(2,2)\end{array}$ & $\begin{array}{c}8 \\
(3,1)\end{array}$ & $\begin{array}{c}4 \\
(1,3)\end{array}$ & $\begin{array}{c}6 \\
(1,9)\end{array}$ & $\begin{array}{c}0 \\
(0)\end{array}$ & $\begin{array}{c}2 \\
(0,6)\end{array}$ & $\begin{array}{c}2 \\
(0,6)\end{array}$ & $\begin{array}{c}6 \\
(1,7)\end{array}$ & $\begin{array}{c}6 \\
(1,6)\end{array}$ & $\begin{array}{c}6 \\
(1,6)\end{array}$ & $\begin{array}{c}4 \\
(1,1)\end{array}$ \\
\hline
\end{tabular}

${ }^{1}$ Promedio \pm DE. ALa edad de los años 2010 y 2011 es mayor a los demás años ( $\left.p<0,05\right)$. DLa edad de los años 2015,2016 y 2017 fue menor a los demás años $(p<0,05)$.

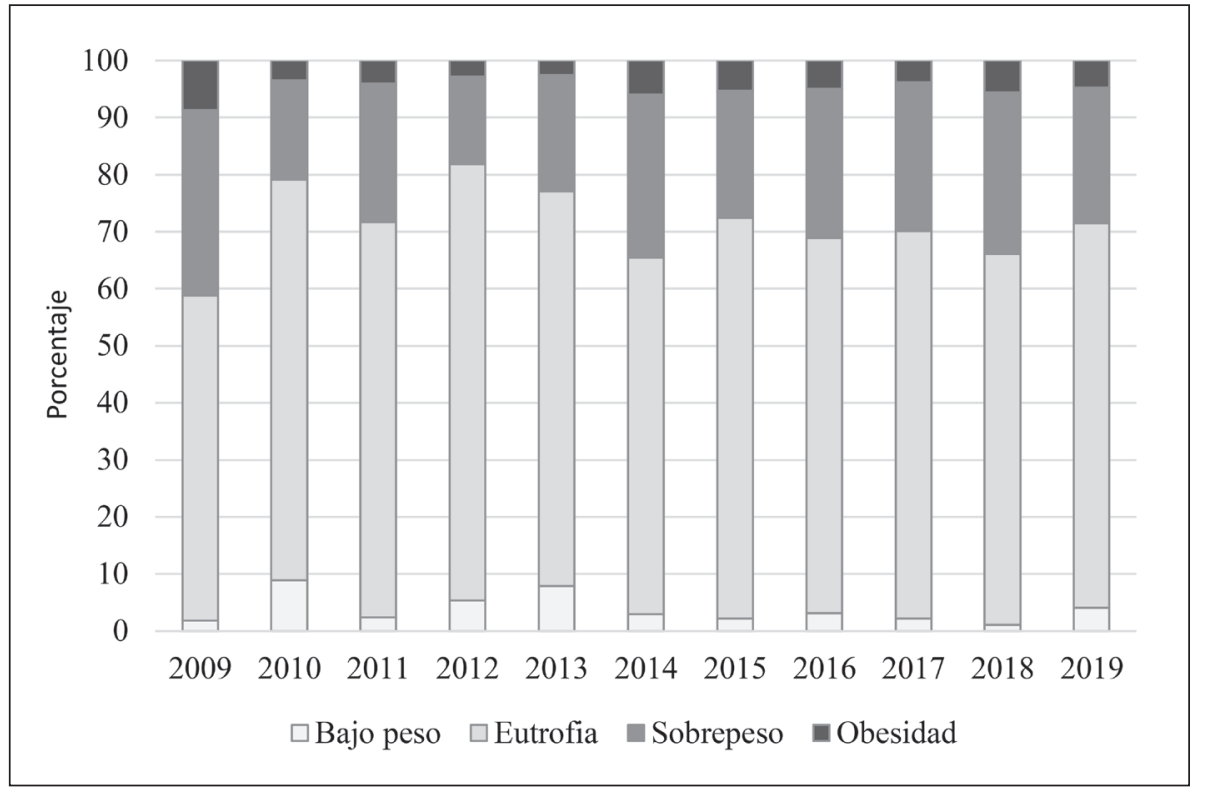

Figura 1. Evolución del estado nutriciona en lactantes y preescolares asistentes a las salas cuna y jardines infantiles de la Universidad Católica de Chile: frecuencias en el mes de marzo de los años 2009 al 2019*.

*Prevalencia de obesidad (Test $\left.\chi^{2}, p=0,038\right)$ La prevalencia de obesidad fue mayor el año 2009 a los años: 2010, 2012, 2013, 2017 $(p<0,05)$ y límite a $2019(p=0,05)$.

* Prevalencia de obesidad + sobrepeso (Test $\chi^{2}, p=0,000$ ). La prevalencia de ambos en conjunto fue mayor el año 2009 a todos demás años, excepto a 2014 y 2018.

Tabla 2. Evolución de los índices antropométricos de los lactantes y preescolares asistentes a los jardines infantiles PUC (marzo de 2009 a marzo de 2019)

\begin{tabular}{ccccccccccccc}
\hline Año & 2009 & 2010 & 2011 & 2012 & 2013 & 2014 & 2015 & 2016 & 2017 & 2018 & 2019 \\
\hline N & 267 & 259 & 297 & 320 & 319 & 336 & 319 & 347 & 370 & 357 & 319 \\
z P/T & $0,84^{\mathrm{A}}$ & $0,32^{\mathrm{C}}$ & $0,59^{\mathrm{B}}$ & $0,36^{\mathrm{C}}$ & $0,36^{\mathrm{C}}$ & $0,65^{\mathrm{B}}$ & $0,55^{\mathrm{B}}$ & $0,60^{\mathrm{B}}$ & $0,59^{\mathrm{B}}$ & $0,67^{\mathrm{B}}$ & $0,55^{\mathrm{B}}$ \\
& $\pm 0,94$ & $\pm 0,91$ & $\pm 0,87$ & $\pm 0,88$ & $\pm 0,88$ & $\pm 0,88$ & $\pm 0,84$ & $\pm 0,87$ & $\pm 0,79$ & $\pm 0,80$ & $\pm 0,87$ \\
\multirow{2}{*}{ Z T/E } & $-0,36$ & $0,03^{\mathrm{B}}$ & $-0,25$ & $-0,11^{\mathrm{B}}$ & $-0,11$ & $-0,26$ & $-0,36$ & $-0,41$ & $-0,47$ & $-0,30$ & $-0,32$ \\
& $0,96 \pm$ & $\pm 1,08$ & $\pm 0,98$ & $\pm 0,98$ & $\pm 0,96$ & $\pm 0,90$ & $\pm 0,91$ & $\pm 0,94$ & $\pm 0,91$ & $\pm 0,90$ & $\pm 0,9$ \\
\hline
\end{tabular}

zP/T: Puntaje z para el índice Peso/Talla. zT/E: Puntaje z para el índice Talla/Edad (Curvas OMS 2006), expresados como promedio \pm DE. ${ }^{A} z P / T 2009$ fue mayor a $z P / T$ de cada uno de los otros años $(p<0,001)$. AzT/E 2010 y 2012 fueron mayores a los otros años, excepto 2013 $(p<0,005)$, test ANOVA y Fisher. 


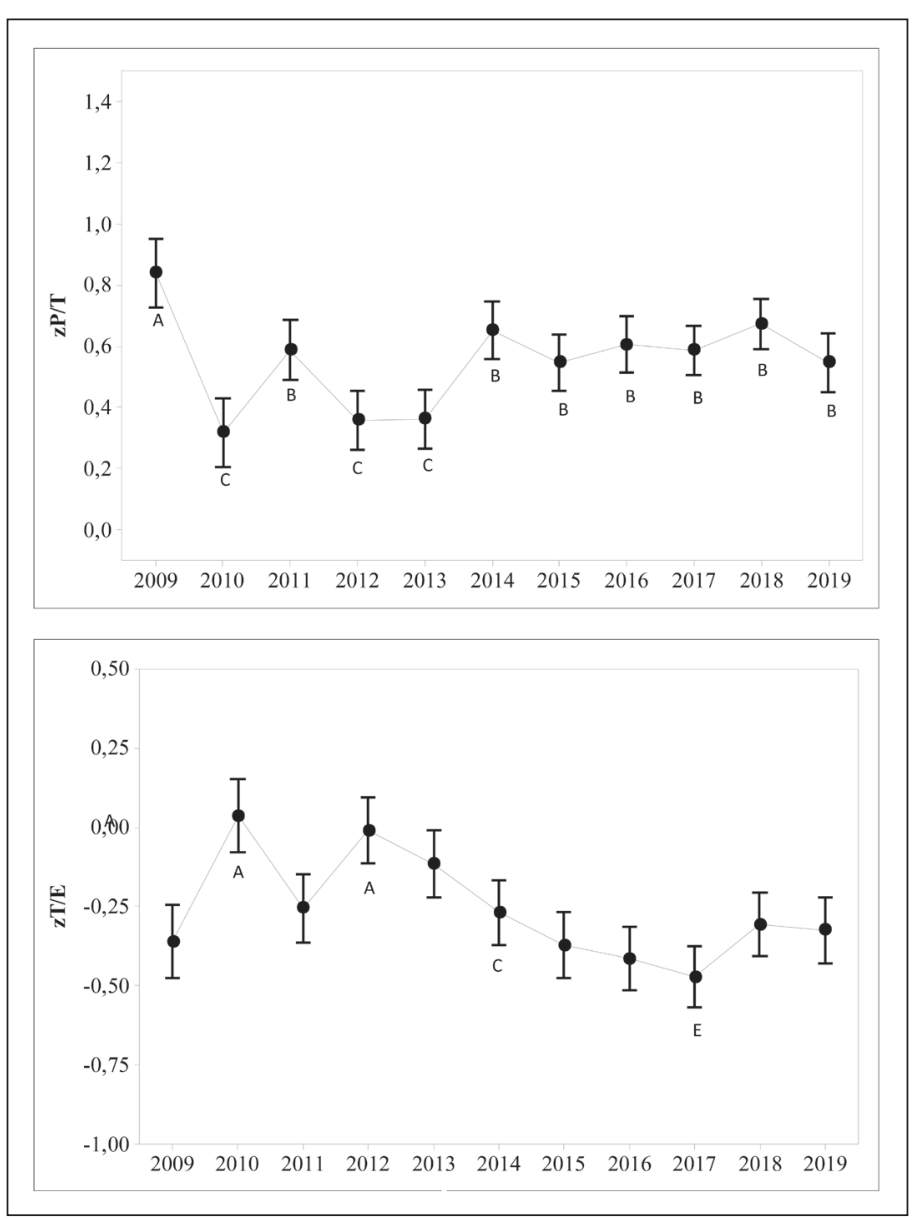

Figura 2. Evolución de los puntajes zP/T y zT/E promedio en lactantes y preescolares asistentes a los centros de Educación Inicial UC, entre 2009 y 2019*. $A_{z P / T} 2009$ fue mayor a $z P / T$ de cada uno de los otros años $(p<0,001)$. ${ }^{A} z T / E$ 2010 y 2012 fueron mayores a los otros años, excepto 2013 ( $p<0,005$ ), test ANOVA y Fisher.

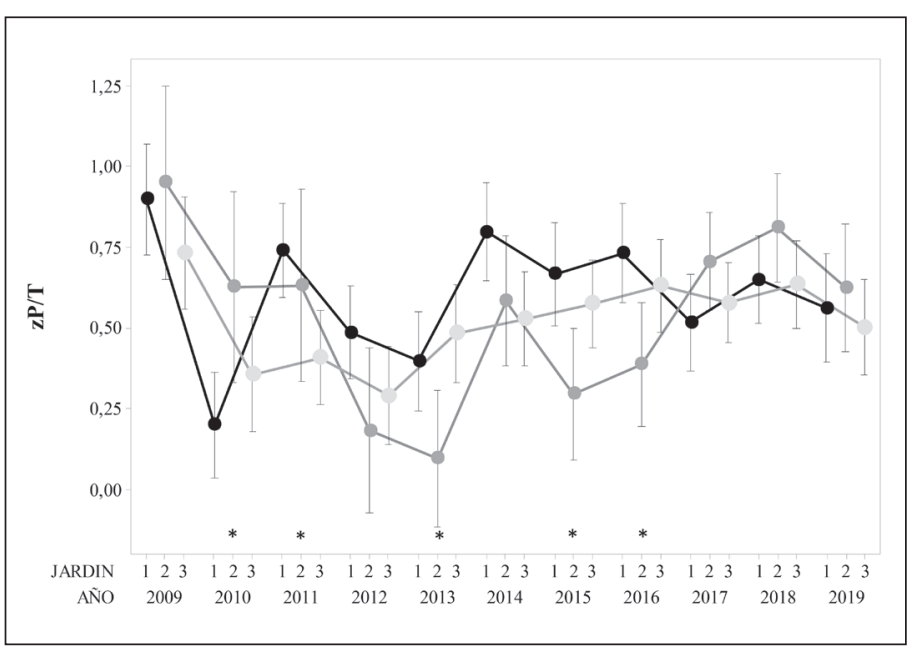

Figura 3. Puntaje zP/T en lactantes y preescolares asistentes a tres centros de Educación Inicial UC, entre 2009 y 2019.

zP/T: Puntaje z para Peso/Talla (Referencia OMS 2006). 1, 2, 3: Centros de Educación Inicial UC. *Diferencia entre al menos dos de los centros en cada año, (ANOVA, $p<0,005$ ).
Al comparar los diferentes centros según el zP/T, se observó una tendencia similar hacia la mejoría, aunque con heterogeneidad los primeros años; hubo mayor $\mathrm{zP} / \mathrm{T}$ en un centro en que $68 \%$ de los padres realizaba trabajo administrativo y menor $\mathrm{zP} / \mathrm{T}$ en otro en que $80 \%$ realizaba trabajo profesional o académico, sin embargo, la diferencia solo fue significativa el año 2016 y los últimos tres años los tres centros fueron comparables (figura 3).

No se observó diferencia en las frecuencias de sobrepeso u obesidad ni en $\mathrm{zP} / \mathrm{T}$ según el género. En cuanto a la edad, hubo tendencia a mayor proporción de malnutrición por exceso (obesidad + sobrepeso) en los niños mayores de dos años, pero la diferencia fue significativa solamente en los años 2009 y 2014. También el zP/T fue más elevado en los mayores de dos años durante el primer período, aunque sin diferencia en los últimos tres años (figura 4).

En la figura 5 se muestra la frecuencia de los diferentes estados nutricionales en los centros UC en marzo de 2019, junto a las últimas prevalencias nacionales disponibles en los centros de la Junta Nacional de Jardines Infantiles (JUNJI) en 61.000 párvulos en $2019^{12}$, de la Fundación Integra en 92.000 párvulos en $2018^{13}$ y la reportada por el Ministerio de Salud, MINSAL, en 922.000 menores de 6 años bajo control en el sistema público en $2017^{2}$. Se observa una menor y significativa prevalencia de malnutrición por exceso en los centros HaViSa UC.

El análisis de los casos nuevos que ingresan cada año a los centros PUC demostró que, como era esperable, su edad era menor que la de los que ya están asistiendo a los centros, con 19,1 $\pm 12,7$ meses los nuevos vs 30,6 $\pm 11,7$ meses los antiguos $(\mathrm{p}=0,000)$. Los niños nuevos tuvieron mayor frecuencia de malnutrición por déficit y menor frecuencia de malnutrición por exceso, diferencia significativa sólo los años 2010 y 2011 (Chi2, $\mathrm{p}=0,001$ y p $=0,000)$. Sin embargo, el zP/T de los nuevos aumentó entre 2010 y 2019, desde $0,39 \pm 0,85$ a $0,59 \pm 0,82$, con diferencia de $0,20 \pm 0,08$ (IC: 0,0415 ; $0,3578$ y $\mathrm{p}=0,013)$.

\section{Discusión}

Este trabajo muestra los resultados de un programa de prevención de obesidad aplicado en tres centros de educación inicial durante un período de diez años, que se asoció a una disminución de la malnutrición por exceso. Esta disminución del sobrepeso en $27 \%$ y de la obesidad en $45 \%$, con cambio de la prevalencia en $-8,76 \%$ y $-3,91 \%$ respectivamente, ha sido significativa y mantenida, lo cual es exitoso, considerando que en Chile la prevalencia del exceso de peso ha aumentado en los menores de 6 años durante este período, en $+0,74$ y $+2,28 \%$ respectivamente ${ }^{2}$. 


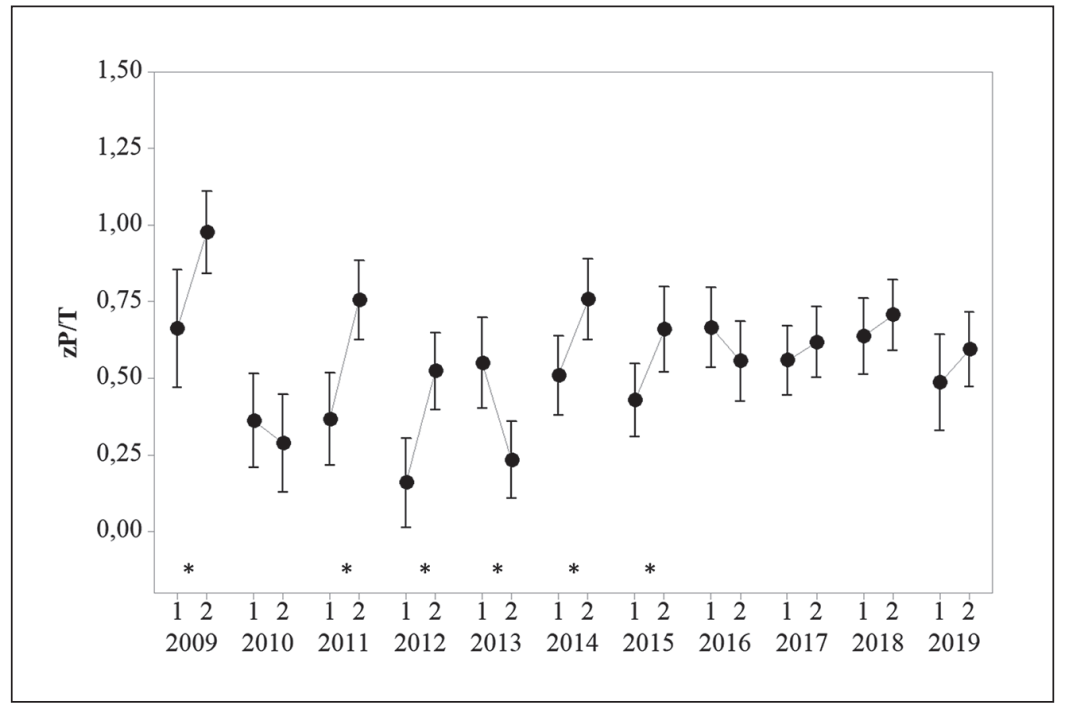

Figura 4. Promedios del puntaje zP/T según la edad, en asistentes a salas cuna y jardines infantiles UC, entre 2009 y 2019.

zP/T: Puntaje z para Peso/Talla (Referencia OMS 2006). 1: Edad menor a dos años, 2: Edad igual o mayor a dos años. Las líneas unen los promedios de ambos grupos de edad para cada año. ${ }^{*}$ Diferencias significativas $(p<0,05)$.

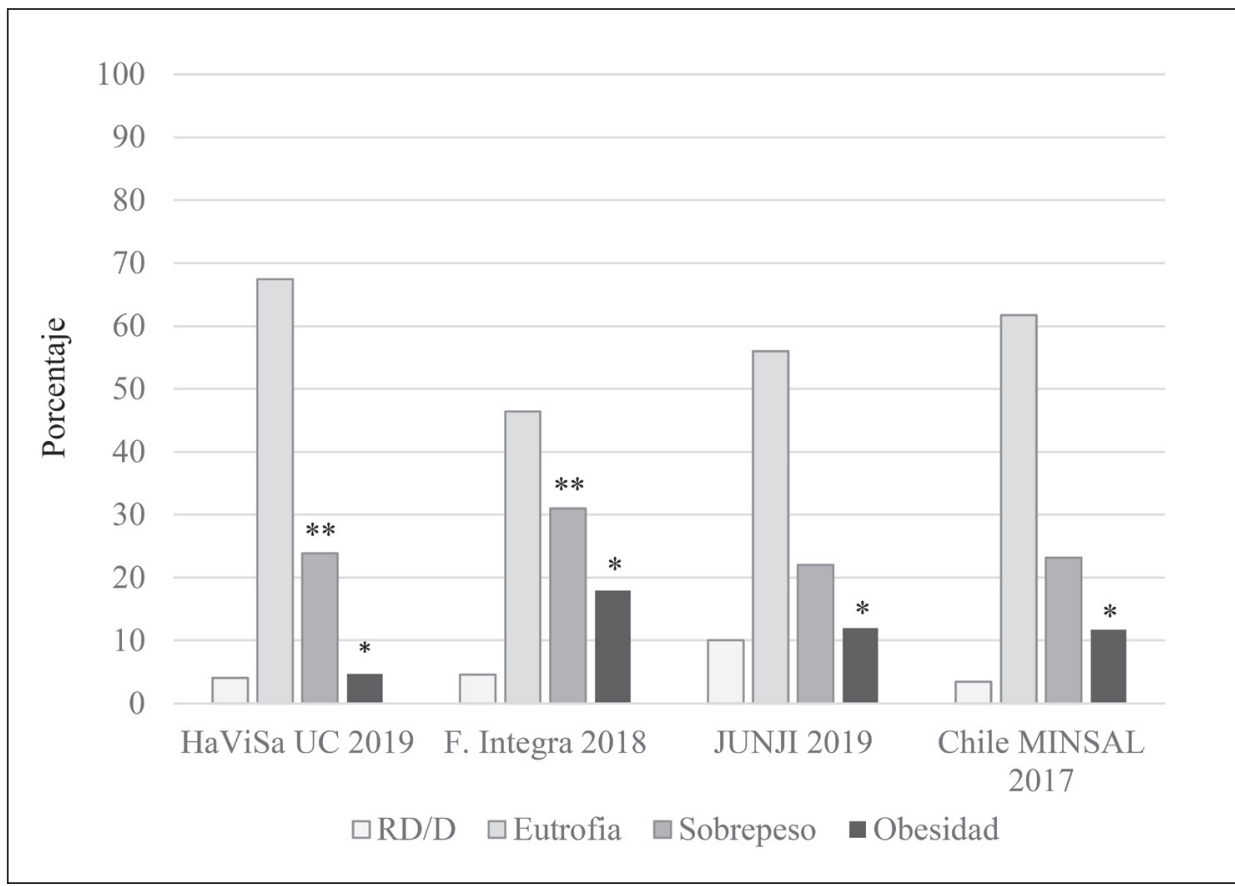

Figura 5. Comparación de las prevalencias de los estados nutricionales en lactantes y preescolares en las salas cuna y jardines infantiles UC en 2019, con los disponibles a nivel nacional entre 2017 y 2019.

* Prevalencia de obesidad en centros HaVisa UC fue menor a los otros tres reportes $(p=0,000)$. **Prevalencia de sobrepeso HaViSa UC fue menor a F. Integra $(p=0,005)$.
El programa involucró acciones destinadas a mejorar tanto los hábitos de alimentación como los de actividad física en forma conjunta, lo cual, con evidencia moderada, tiene mejor rendimiento en los niños menores de 6 años ${ }^{15}$. También se encuentra en sintonía con estudios recientes de prevención de la obesidad a partir del primer año de vida ${ }^{21}$, lo cual favorece la continuidad y un mejor resultado de estrategias aplicadas después. Se trata de una intervención multicomponente, que involucra diferentes medidas. La mayoría de los cambios implementados fueron de tipo estructural, es decir, cuyo mecanismo de acción es independiente de las acciones o deseos del individuo o de su familia.
Ejemplos de ello son: el cambio de los jarros de jugo por bebederos de agua, o de la colación antes enviada desde el hogar por una fruta entregada por el centro, así como la disminución del tamaño de las porciones de la comida o el cambio a leche descremada en los mayores de dos años con obesidad. También se realizaron acciones dependientes de la voluntad o acción del individuo o familia ("agentic" en idioma inglés), que corresponden a actividades educativas, como por ejemplo: guías y recomendaciones enviadas al hogar, envío de "placemat" con el tamaño de las porciones de comida o los talleres educacionales. Otras correspondieron a acciones mixtas, que involucran cambios 
ambientales, pero sobre las cuales el sujeto aún tiene poder de decisión (ejemplo: artefactos de ejercicio en el jardín, o la incorporación de la clase de educación física). Debido a que los niños permanecen durante una proporción importante de su vida diaria en los centros, las medidas estructurales son probablemente las más efectivas a corto plazo y las acciones educativas, la mayoría de tipo mixto, a mediano y largo plazo.

Una consideración necesaria es que los programas de prevención no incrementen las disparidades, es decir, que favorezcan especialmente a los grupos que presentan menores factores de riesgo $\mathrm{y}$, por tanto, menor prevalencia de obesidad ${ }^{45}$. La respuesta homogénea en los últimos tres años de los tres centros en nuestra muestra, con diferente representación socioeconómica, es un indicador indirecto de ausencia de este efecto. Otra consecuencia no deseada puede ser un aumento de los niños con bajo peso, pero aunque en este período de 10 años hubo un leve incremento de la malnutrición por déficit, desde de 1,87 a 4,08\% (+2,21\%), éste fue comparable al aumento de $2,7 \%$ a $4,4 \%(+1,7 \%)$ reportado en los menores de 6 años en control en el sistema público chileno ${ }^{2}$. Este subgrupo fue monitorizado especialmente, y correspondió casi en su totalidad a niños en rango de "riesgo a desnutrir", como prematuros, gemelares o niños con patologías crónicas, así como una proporción de niños con delgadez constitucional, situación fisiológica en la cual no se recomienda la sobrealimentación.

La principal fortaleza de este programa es su permanencia en el tiempo, medida fundamental para mantener los resultados favorables, ya que frente al ambiente altamente obesogénico, cuya mejoría es lenta, se requiere del estímulo constante de hábitos de vida saludable. También es una ventaja importante su aplicación durante el período inicial de la vida, cuando el cambio de hábitos y la adherencia familiar están favorecidos $^{46}$. Es también fortaleza el que sea multicomponente, incluyendo cambios de la alimentación y la actividad física y que abarque a todos los actores desde el punto de vista educativo: los niños, el personal del jardín y la familia. La educación continua de los profesionales y del personal de los centros es fundamental, así como hacer consciente en ellos la necesidad de un buen autocuidado.

La ausencia de un grupo control constituye una limitación de este estudio, así como el análisis de cortes anuales, en distintos grupos de niños. Sin embargo, los buenos resultados frente a la estabilidad y larga duración del programa contrastan con lo ocurrido a nivel nacional con esta pandemia. Para evaluar los resultados de modo longitudinal, se encuentra en desarrollo un análisis de las trayectorias de crecimiento de los niños que completaron una estadía de al menos dos años en los centros.
La menor representatividad de esta muestra es una limitación, al estar constituida por hijos de personal administrativo, docentes y alumnas, de modo que la proporción de familias de menor nivel socioeconómico es más baja que la de la población general, factor de riesgo de obesidad ${ }^{47}$. A pesar de ello, al inicio las frecuencias de obesidad y sobrepeso eran similares a las prevalencias nacionales, logrando una caída inicial importante que, con fluctuaciones menores se ha mantenido estable, mientras han aumentado en la población general.

La mejor respuesta inicial fue probablemente debida a la implementación rápida y efectiva de los cambios alimentarios, el ingreso de lactantes con menor sobrepeso u obesidad en 2010 y 2011, que se incorporaron de inmediato al programa, así como al egreso de niños con exceso de peso de mayor duración y posiblemente con menor reversibilidad. El ascenso posterior a 2013 de la malnutrición por exceso (aunque a un nivel menor que el inicial) puede deberse al ingreso anual de niños con mayor zP/T a partir de ese año, que, aunque no tenían mayor frecuencia de diagnóstico de obesidad o sobrepeso a su llegada, ascendían con mayor facilidad a estas categorías. También influye la necesidad de educación permanente del entorno y de actualización de las estrategias implementadas, frente a un ambiente externo que mantiene numerosos factores de riesgo obesogénicos.

Un aspecto por considerar son las diversas intervenciones que se fueron sumando y adaptando en el tiempo al programa HaViSa, lo cual no permite adjudicar a medidas específicas el efecto favorable logrado. Pero, por un lado, en los cambios implementados se aplicó la mejor evidencia disponible $e^{15,48}$ y por otro, fue necesario enfrentar nuevas necesidades de un proyecto que se transformó en programa permanente.

En cuanto a aspectos por mejorar, debemos aumentar la actividad física; el próximo desafío será también conocer el nivel de ésta en el hogar y tomar medidas acordes a los resultados, estimulando la disminución del sedentarismo y especialmente del tiempo de pantallas ${ }^{40-43}$. Se requiere también que los logros alcanzados no se pierdan al ingresar al colegio, momento crucial de aumento de la independencia e influencia de los pares y el ambiente.

Durante este período pensamos que el efecto favorable de la información cercana y regular a los padres ha sido de utilidad para comunicar a tiempo la aceleración de la ganancia ponderal y entregar herramientas de mejora cuando el niño es menor y/o tiene menor grado de exceso de peso. Un factor que influye en el empeoramiento nutricional es la disminución o ausencia de controles de salud regulares en la edad escolar, que afecta la percepción del problema ${ }^{49}$.

Aunque corresponde a una intervención focalizada, 
este programa ha sido exitoso en disminuir la obesidad en esta muestra de lactantes y preescolares. Reconocemos que se requieren políticas de salud poblacionales coordinadas, que favorezcan ambientes saludables en alimentación y actividad física, y que éstas son más costo-efectivas. Sin embargo, debe haber un compromiso permanente en todos los niveles de la sociedad. Se espera a través de este reporte, entregar herramientas preventivas que sean útiles a las instituciones públicas que reciben a niños de esta edad, para desarrollar esfuerzos conjuntos y lograr ser establecimientos educacionales realmente promotores de la salud.

En conclusión, este reporte muestra que a 10 años de la implementación del programa de prevención HaViSa se produjo en estos tres centros de educación inicial, una disminución significativa y permanente de la obesidad y el sobrepeso.

\section{Responsabilidades Éticas}

Protección de personas y animales: Los autores declaran que los procedimientos seguidos se conformaron a las normas éticas del comité de experimentación humana responsable y de acuerdo con la Asociación Médica Mundial y la Declaración de Helsinki.

Confidencialidad de los datos: Los autores declaran que han seguido los protocolos de su centro de trabajo sobre la publicación de datos de pacientes.

Derecho a la Privacidad y Consentimiento Informado: Este estudio ha ido aprobado por el Comité de Éti- ca de Investigación correspondiente, quien de acuerdo a las características del estudio ha eximido el uso del Consentimiento Informado.

\section{Conflicto de intereses}

Los autores declaran no tener conflicto de intereses.

\section{Financiamiento}

El Programa HaViSa ha sido financiado por el Departamento de Beneficios al Personal de la Pontificia Universidad Católica de Chile.

\section{Agradecimientos}

Agradecemos a las educadoras, auxiliares de párvulos, personal de los servicios de alimentación y administrativos, de los Centros de Educación Inicial de la Pontificia Universidad Católica de Chile, por su colaboración en llevar a cabo el trabajo de campo, desde el inicio del Programa HaViSa en marzo de 2009. A las autoridades de la Universidad y a la Dirección de Beneficios del Personal por su apoyo permanente. A la Dirección de Informática y la Dirección de Diseño por su ayuda experta. A los padres de los niños que asisten, por la buena recepción de los informes, material educativo y diferentes actividades que se envían al hogar, pero, sobre todo, por comprometerse con la salud de sus hijas e hijos. A los niños que han pasado este tiempo importante de su vida en los centros, por ser siempre terreno fértil de aprendizaje.

\section{Referencias}

1. ENCUESTA NACIONAL DE SALUD 2016-2017. https://www.minsal.cl/wpcontent/uploads/2017/11/ENS-2016-17. pdf

2. Estado Nutricional Niños y Niñas Menores de 6 años y Mujeres Gestantes. Población Bajo Control en el Sistema de Salud de Chile. Censo diciembre de 2017. http://www.bibliotecaminsal.cl/vigilanciadel-estado-nutricional-de-la-poblacionbajo-control-y-de-la-lactancia-maternaen-el-sistema-publico-de-salud-de-chile/

3. JUNAEB, Mapa Nutricional 2018. https:// www.junaeb.cl/mapa-nutricional

4. Guo SS, Roche AF, Chumlea WC, Garner JD, Siervogel RM. The predictive value of childhood body mass index values for overweight at age 35 y.o. Am J Clin Nutr. 1994;59: 810-9.

5. Whitaker RC, Wright JA, Pepe MS,
Seidel KD, Dietz WH. Predicting obesity in young adulthood from childhood and parental obesity. N Engl J Med. 1997;25;337:869-73.

6. Sharma V, Coleman S, Nixon J, et al. A systematic review and meta-analysis estimating the population prevalence of comorbidities in children and adolescents aged 5 to 18 years. Obes Rev. 2019;20:1341-9.

7. Mead E, Brown T, Rees K, et al. Diet, physical activity and behavioral interventions for the treatment of overweight or obese children from the age of 6 to 11 years. Cochrane Database Syst Rev. 2017;6:CD012651.

8. Barja S, Núñez E, Velandia S, Urrejola P, Hodgson MI. Adherencia y efectividad a mediano plazo del tratamiento de la obesidad infantil. Rev Chil Pediatr. 2005;76:151-58.

9. Moraga F, Rebollo MJ, Bórquez P,
Cáceres J, Castillo C. Tratamiento de la obesidad infantil: Factores pronósticos asociados a una respuesta favorable. Rev Chil Pediatr 2003;74:374-80.

10. Must A, Jacques PF, Dallal GE, Bajema CJ, Dietz WH. Long term morbidity and mortality of overweight adolescents: a following of the Harvard Growth Study of 1992 to 1935 . N Engl J of Med. 1992;327:1350-55.

11. Reilly JJ, Kelly J. Long-term impact of overweight and obesity in childhood and adolescence on morbidity and premature mortality in adulthood: systematic review. Int J Obes (Lond). 2011;35:891-8.

12. Evaluación nutricional 2019. Junta nacional de jardines infantiles, JUNJI, Región Metropolitana. Ministerio de Educación. Chile.

13. Reporte 2018. Situación nutricional de niños y niñas, Fundación INTEGRA http://www.integra.cl/wp-content/ 
uploads/2019/05/REPORTE_2018_ FINAL2.pdf

14. Barlow SE, and the Expert Committee. Expert Committee Recommendations Regarding the Prevention, Assessment and Treatment of Child and Adolescent Overweight and Obesity: Summary Report. Pediatrics. 2007;120:S164-S192.

15. Brown T, Moore TH, Hooper L, et al. Interventions for preventing obesity in children. Cochrane Database Syst Rev. 2019;7(7):CD001871.

16. Hoelscher DM, Kirk S, Ritchie L, Cunningham-Sabo L. Academy Positions Committee. Position of the Academy of Nutrition and Dietetics: interventions for the prevention and treatment of pediatric overweight and obesity. J Acad Nutr Diet. 2013;113:1375-94.

17. Steinberger J, Daniels SR, Hagberg N, et al. Cardiovascular Health Promotion in Children: Challenges and Opportunities for 2020 and Beyond: A Scientific Statement from the American Heart Association. Circulation. 2016;134:e236-e255.

18. Pérez-Escamilla R, Lutter CK, RabadanDiehl C, et al. Prevention of childhood obesity and food policies in Latin America: from research to practice. Obes Rev. 2017;18 Suppl 2:28-38.

19. Cushing CC, Brannon EE, Suorsa KI, Wilson DK. Systematic Review and Meta-Analysis of Health Promotion interventions for children and adolescents using an ecological framework. J Pediatr Psychol. 2014;39:949-62.

20. Pérez Escanilla and Kac G. Childhood obesity prevention: a life-course framework. Int J Obes. 2013;3:S3-S5.

21. Volger S, Rigassio Radler D, RothpletzPuglia P. Early childhood obesity prevention efforts through a life course health development perspective: A scoping review. PLoS One. 2018;13:e0209787.

22. Hu Y, He JR, Liu FH, et al. Effectiveness of a Kindergarten-Based Intervention for Preventing Childhood Obesity. Pediatrics. 2017;140; pii:e20171221.

23. Hassink SG. Early Child Care and Education: A Key Component of Obesity Prevention in Infancy. Pediatrics. 2017;140:1-3.

24. O'Brien M, Nader PR, Houts RM, et al. and the NICHD Early Child Care Research Network. The Ecology of Childhood Overweight: A 12-Year Longitudinal Analysis. Int J Obes (Lond). 2007;31:1469-78.

25. Kain J, Uauy R, Concha F, et al. Schoolbased obesity prevention interventions for Chilean children during the past decades: lessons learned. Adv Nutr. 2012;3:616S-21S.
26. Vio F, Salinas J, Montenegro E, González CG, Lera L. Efecto de una intervención educativa en alimentación saludable en profesores y niños preescolares y escolares de la región de Valparaíso, Chile. Nutr Hosp. 2014;29:1298-304.

27. The WHO Child Growth Standards. https://www.who.int/childgrowth/en/

28. Patro-Goł b B, Zalewski BM, Kołodziej M, et al. Nutritional interventions or exposures in infants and children aged up to 3 years and their effects on subsequent risk of overweight, obesity and body fat: a systematic review of systematic reviews. Obes Rev. 2016;17:1245-57.

29. Arenz S, Ruckerl R, Koletzko B, von Kries $\mathrm{R}$ : Breast-feeding and childhood obesity - a systematic review. Int J Obes Relat Metab Disord. 2004;28:1247-56.

30. Horta bl, Bahl l, Martines JC, Victora CG. Evidence on the long-term effects of breastfeeding: systematic review and meta-analyses. World Health Organization 2007. ISBN 978924 159523 0. WHO Press, World Health Organization.

31. Oyarzún MF, Barja S, Domínguez MA, Villarroel L, Arnaiz P, Mardones F. Lactancia materna, obesidad y síndrome metabólico en la edad escolar. Rev Chil Pediatr. 2018;89:173-81.

32. Guía de alimentación del niño(a) menor de 2 años y guía de alimentación hasta la adolescencia. Ministerio de Salud, Chile. http://www.crececontigo.gob. cl/wp-content/uploads/2016/01/Guiaalimentacion-menor-de-2.pdf

33. Butte NF. Impact of infant feeding practices on childhood obesity. J Nutr. 2009;139:412S-6S.

34. Gidding S, Dennison BA, Birch LL, et al. Dietary Recommendations for Children and Adolescents: A Guide for Practitioners: Consensus Statement from the American Heart Association. Endorsed by the American Academy of Pediatrics. Circulation 2005;112: 2061-75.

35. Laving AR, Hussain SR, Atieno DO. Overnutrition: ¿Does Complementary Feeding Play a Role? Ann Nutr Metab. 2018;73(suppl 1):15-8.

36. Scharf RJ, DeBoer MD. Sugar-Sweetened Beverages and Children's Health. Annu Rev Public Health. 2016;37:273-93.

37. Fidler Mis N, Braegger C, Bronsky $\mathrm{J}$, et al. Sugar in Infants, Children and Adolescents: A Position Paper of the European Society for Paediatric Gastroenterology, Hepatology and Nutrition Committee on Nutrition. J Pediatr Gastroenterol Nutr. 2017;65:68196.

38. Dougkas A, Barr S, Reddy S, Summerbell CD. A critical review of the role of milk and other dairy products in the development of obesity in children and adolescents. Nutr Res Rev. 2019;32:10627.

39. Golden NH, Schneider M, Wood C; Committee on nutrition; Committee on adolescence; Section on obesity. Preventing Obesity and Eating Disorders in Adolescents. Pediatrics. 2016;138:e1e10.

40. Council on Sports Medicine and Fitness; Council on School Health. Active healthy living: prevention of childhood obesity through increased physical activity. Pediatrics. 2006;117:1834-42.

41. De Ferranti SD, Steinberger J, Ameduri $\mathrm{R}$, et al. Cardiovascular Risk Reduction in High-Risk Pediatric Patients: A Scientific Statement from the American Heart Association. Circulation. 2019;139:e603-e634.

42. Steinberger J, Daniels SR, Hagberg $\mathrm{N}$, et al. Cardiovascular Health Promotion in Children: Challenges and Opportunities for 2020 and Beyond: A Scientific Statement from the American Heart Association. Circulation. 2016;134:e236-e255.

43. Engberg E, Figueiredo RAO, Rounge TB, Weiderpass E6 Viljakainen H. Heavy screen users are the heaviest among 10,000 children. Sci Rep. 2019;9:11158.

44. Zemel BS, Pipan M, Stallings VA, et al. Growth Charts for Children with Down Syndrome in the United States. Pediatrics. 2015;136:e1204-11.

45. Venturelli F, Ferrari F, Broccoli S, et al. The effect of Public Health/Pediatric Obesity interventions on socioeconomic inequalities in childhood obesity: A scoping review. Obes Rev. 2019;20:172039.

46. Campbell KJ, Hesketh KD, McNaughton SA, et al. The extended Infant Feeding, Activity and Nutrition Trial (InFANT Extend) Program: a cluster-randomized controlled trial of an early intervention to prevent childhood obesity. BMC Public Health. 2016;16:166.

47. Wu S, Ding Y, Wu F, et al. Socioeconomic position as an intervention against overweight and obesity in children: a systematic review and metaanalysis. Sci Rep. 2015;5:11354.

48. Koletzko B, Brands B, Grote V, et al. Early Nutrition Programming Project. LongTerm Health Impact of Early Nutrition: The Power of Programming. Ann Nutr Metab. 2017;70:161-69.

49. García T Andrea, Aspillaga C, CruzatMandich C, Barja S. Recomendaciones de padres de niños obesos en tratamiento para el equipo de salud: estudio cualitativo. Rev Chil Pediatr. 2017;88: 495-501. 